\title{
Ferritin levels and COVID-19
}

Suggested citation Vargas-Vargas M and Cortés-Rojo C. Ferritin levels and COVID-19. Rev Panam Salud Publica. 2020;44:e72. https://doi. org/10.26633/RPSP.2020.72

\section{To the Editor,}

Ferritin is a key mediator of immune dysregulation, especially under extreme hyperferritinemia, via direct immune-suppressive and pro-inflammatory effects, contributing to the cytokine storm $^{1}$. It has been reported that fatal outcomes by COVID-19 are accompanied by cytokine storm syndrome, thereby it has been suggested that disease severity is dependent of the cytokine storm syndrome ${ }^{2}$. Many individuals with diabetes exhibit elevated serum ferritin levels ${ }^{3-5}$, and it is known that they face a higher probability to experience serious complications from COVID-196. On this basis, we briefly review evidence supporting the hypothesis that ferritin levels might be a crucial factor influencing the severity of COVID-19.

In one study with 20 COVID-19 patients, it was found that individuals with severe and very severe COVID-19 exhibited increased serum ferritin level, being serum ferritin in the very severe COVID-19 group significantly higher than in the severe COVID-19 group (1006.16 ng/ml [IQR: 408.265-1988.25] vs $291.13 \mathrm{ng} / \mathrm{ml}$ [IQR: 102.1-648.42], respectively) ${ }^{7}$. In agreement with this, another study revealed that in patients who died by COVID-19, ferritin levels were high upon hospital admission and throughout the hospital stay. The median values of serum ferritin levels after day 16 of hospitalization exceeded the upper limit of detection in these patients, suggesting that ferritin levels increased non-stop ${ }^{8}$. Also, Chen et al. analyzed the clinical characteristics of 99 patients, in which 63 of them had serum ferritin way above of the normal range ${ }^{9}$. Elevated ferritin levels were found also in autopsies of 12 patients whose cause of death was SARS-CoV-2 infection ${ }^{10}$. An analysis of the peripheral blood of 69 patients with severe COVID-19 revealed elevated levels of ferritin compared with patients with non-severe disease. Therefore, it was concluded that serum ferritin levels were closely related to the severity of COVID-1911. Finally, laboratory findings in patients with severe COVID-19 showed data consistent with cytokine storm involving elevated inflammatory markers, including ferritin, which has been associated with critical and life-threatening illness ${ }^{12}$.

A possible strategy to decrease ferritin levels might be the treatment with iron chelators. Deferoxamine may be a good candidate, since is a non-toxic iron chelator clinically approved by the FDA and is effective for long-term iron chelation therapy in beta-thalassemia and other maladies involving iron overload ${ }^{13}$. Manipulations decreasing dietary iron should be also considered as they have been shown to modify serum ferritin levels $^{14,15}$. Thus we hypothesized that this might reduce the exacerbation of COVID-19, specially in individuals with morbidities cursing with elevated ferritin levels such as diabetes ${ }^{3-5}$.

Disclaimer. Authors hold sole responsibility for the views expressed in the manuscript, which may not necessarily reflect the opinion or policy of the RPSP/PAJPH or the Pan American Health Organization (PAHO).

Manuel Vargas-Vargas,

Christian Cortés-Rojo

Universidad Michoacana de San Nicolás de Hidalgo, Morelia, México

$\triangle$ Manuel Vargas-Vargas, manuelalejandrovargas@hotmail.com

\section{REFERENCES}

1. Abbaspour N, Hurrell R, Kelishadi R. Review on iron and its importance for human health. Research J Med Sci. 2014;19(2): 164-74.

2. Huang C, Wang Y, Li X, Ren L, Zhao J, Hu Y, et al. Lancet. 2020; 395:497-506.

3. Khalil UA, Seliem FO, Alnahal A, Awad M, Sadek AM, Fawzy MS. Association of serum ferritin with insulin resistance in offsprings of type 2 diabetes. Egypt J Intern Med. 2018;30:13-7.

4. Momeni A, Behradmanesh MS, Kheiri S, Abasi F. Serum ferritin has correlation with $\mathrm{HbA} 1 \mathrm{c}$ in type 2 diabetic patients. Adv Biomed Res. 2015;4:74.

5. Son NE. Influence of ferritin levels and inflammatory markers on $\mathrm{HbA} 1 \mathrm{c}$ in the Type 2 Diabetes mellitus patients. Pak J Med Sci. 2019;35(4):1030-35.
6. American Diabetes Association. How COVID-19 Impacts People with Diabetes. Available at: https://www.diabetes.org/corona virus-covid-19/how-coronavirus-impacts-people-with-diabetes Accessed May 22, 2020

7. Bo Zhou, Jianqing She, Yadan Wang. Utility of Ferritin, Procalcitonin, and C-reactive Protein in Severe Patients with 2019 Novel Coronavirus Disease; 2020. Available at https://doi.org/10.21203/ rs.3.rs-18079/v1 Accessed May 22, 2020

8. Fei Zhou, Ting Yu, Ronghui Du, Guohui Fan, Ying Liu, Zhibo Liu, et al. Clinical course and risk factors for mortality of adult inpatients with COVID-19 in Wuhan, China: a retrospective cohort study. Lancet. 2020;395:1054-62

9. Chen N, Zhou M, Dong X, Qu J, Gong F, Han Y, et al. Epidemiological and clinical characteristics of 99 cases of 2019 novel coronavirus 
pneumonia in Wuhan, China: a descriptive study. Lancet. 2020; 395(10223):507-13.

10. Fox SE, Akmatbekov A, Harbert JL, Li G, Brown JQ, Vander Heide RS. Pulmonary and Cardiac Pathology in Covid-19: The First Autopsy Series from New Orleans. medRxiv 2020.04.06-20050575; doi: https:/ /doi.org/10.1101/2020.04.06.20050575.

11. Tao Liu, Jieying Zhang, Yuhui Yang, Hong Ma, Zhengyu Li, Jiaoyu Zhang, et al. The potential role of IL- 6 in monitoring severe case of coronavirus disease 2019. medRxiv 2020.03.01.20029769; doi: https: / / doi.org/10.1101/2020.03.01.20029769

12. Mehta P, McAuley DF, Brown M, Sanchez E, Tattersall RS, Manson J. COVID-19: consider cytokine storm syndromes and immunosuppression. Lancet. 2020. Available at https://www.thelancet.com/ journals/lancet/article/PIIS0140-6736(20)306280/fulltext Accessed on May 22, 2020

13. Mobarra N, Shanaki M, Ehteram H, Nasiri H, Sahmani M, Saeidi M, et al. A Review on Iron Chelators in Treatment of Iron
Overload Syndromes. Int J Hematol Oncol Stem Cell Res. 2016; 10(4):239-47.

14. Ju SY, Ha AW. Dietary factors associated with high serum ferritin levels in postmenopausal women with the Fifth Korea National Health and Nutrition Examination Survey (KNHANES V), 20102012. Nutr Res Pract. 2016;10(1):81-8.

15. Fleming DJ, Tucker KL, Jacques PF, Dallal GE, Wilson PWF, Wood RJ. Dietary factors associated with the risk of high iron stores in the elderly Framingham Heart Study cohort. Am J Clin Nutr. 2002;76:1375-84.

Manuscript received May 7 2020. Accepted for publication May 22, 2020. Not peer-reviewed. 